\title{
A NOTE CONCERNING BEHAVIOUR OF ITERATED LOGARITHM TYPE
}

\author{
C. C. HEYDE
}

Let $\left\{X_{i}, i=1,2,3, \cdots\right\}$ be a sequence of independent and identically distributed random variables and write $S_{n}=\sum_{i=1}^{n} X_{i}, n \geqq 1$. It is well known that

$$
\operatorname{Pr}\left(\limsup _{n \rightarrow \infty}(2 n \log \log n)^{-1 / 2} S_{n}=1\right)=1
$$

if and only if $E X_{i}=0, E X_{i}^{2}=1$ (Hartman and Wintner [6] obtained the sufficiency part and Strassen [9] the necessity) and the purpose of this note is to clarify the corresponding situation in the case where these moment conditions are violated. It turns out that the oscillation behaviour of normed sums rests essentially on whether or not the summands belong to the domain of partial attraction of the normal distribution.

Firstly, we remark that it has been shown by Freedman (see Strassen [9]) that there exist symmetric random variables $X_{i}$ with $E X_{i}^{2}=\infty$ or even $E\left|X_{i}\right|=\infty$, for which it is possible to choose a monotone sequence $\left\{b_{n}, n=1,2,3, \cdots\right\}$ of positive constants with $b_{n} \rightarrow \infty$ as $n \rightarrow \infty$ such that

$$
\operatorname{Pr}\left(\limsup _{n \rightarrow \infty} b_{n}^{-1} S_{n}=1\right)=1 .
$$

With this in mind, we go on to establish the following theorem.

THEOREM 1. Suppose the $X_{i}$ are such that there exists a monotone sequence of positive constants $\left\{b_{n}, n=1,2,3, \cdots\right\}$ with $b_{n} \rightarrow \infty$ as $n \rightarrow \infty$ for which

$$
\limsup _{n \rightarrow \infty} b_{n}^{-1}\left|S_{n}\right|<\infty
$$

with positive probability. Then, at least one of the following two conditions is satisfied:

(i) $b_{n}^{-1}\left(S_{n}-\operatorname{med} S_{n}\right) \stackrel{\text { a.s. }}{\longrightarrow} 0$.

(ii) The $X_{i}$ belong to the domain of partial attraction of the normal distribution. A necessary and sufficient condition for this is (Lévy $[7$, p. 113])

Received by the editors November 1, 1968. 


$$
\liminf _{u \rightarrow \infty} \frac{u^{2} \operatorname{Pr}\left(\left|X_{1}\right|>u\right)}{\int_{|x| \leqq u} x^{2} d \operatorname{Pr}\left(X_{1} \leqq u\right)}=0 .
$$

("a.s." denotes almost sure convergence; "med" is an abbreviation of median).

Proof. Firstly, since (1) is a tail event on the $S_{1}, S_{2}, \ldots$ process it has probability one using the Hewitt-Savage zero-one law (e.g. Breiman [1, Corollary 3.50]).

Next, suppose that neither (i) nor (ii) are satisfied. From (2), there exists a constant $C>0$ such that

$$
u^{2} \operatorname{Pr}\left(\left|X_{1}\right|>u\right)>C \int_{|x| \leqq u} x^{2} d \operatorname{Pr}\left(X_{1} \leqq \varkappa\right)
$$

for $u>U$. Thus, for $u>U$ and $\eta>1$,

$$
\begin{aligned}
u^{2}\left[\operatorname{Pr}\left(\left|X_{1}\right|>u\right)-\operatorname{Pr}\left(\left|X_{1}\right|>\eta u\right)\right] & \leqq \int_{u<|x| \leqq \eta u} x^{2} d \operatorname{Pr}\left(X_{1} \leqq x\right) \\
& \leqq u^{2} C^{-1} \eta^{2} \operatorname{Pr}\left(\left|X_{1}\right|>\eta u\right),
\end{aligned}
$$

from which we obtain

$$
\operatorname{Pr}\left(\left|X_{1}\right|>u\right) \leqq\left(1+C^{-1} \eta^{2}\right) \operatorname{Pr}\left(\left|X_{1}\right|>\eta u\right) .
$$

Thus, for $n$ sufficiently large we have, when $\epsilon>1$,

$$
\left(1+C^{-1} \epsilon^{2}\right)^{-1} \operatorname{Pr}\left(\left|X_{1}\right|>b_{n}\right) \leqq \operatorname{Pr}\left(\left|X_{1}\right|>\epsilon b_{n}\right) \leqq \operatorname{Pr}\left(\left|X_{1}\right|>b_{n}\right),
$$

and when $\epsilon<1$,

$$
\operatorname{Pr}\left(\left|X_{1}\right|>b_{n}\right) \leqq \operatorname{Pr}\left(\left|X_{1}\right|>\epsilon b_{n}\right) \leqq\left(1+C^{-1} \epsilon^{-2}\right) \operatorname{Pr}\left(|X|>b_{n}\right),
$$

so the convergence (divergence) of $\sum \operatorname{Pr}\left(\left|X_{1}\right|>\epsilon b_{n}\right)$ for some $\epsilon>0$ implies convergence (divergence) for every $\epsilon>0$.

If $\sum \operatorname{Pr}\left(\left|X_{1}\right|>\epsilon b_{n}\right)=\infty$ for every $\epsilon>0$, we have from the BorelCantelli Lemmas that

$$
\operatorname{Pr}\left(\left|X_{n}\right|>\epsilon b_{n} \text { i.o. }\right)=1
$$

for every $\epsilon>0$ ("i.o." is an abbreviation of infinitely often). Furthermore, $\left|S_{n}-S_{n-1}\right|=\left|X_{n}\right| \leqq\left|S_{n}\right|+\left|S_{n-1}\right|$, so that (3) implies $\operatorname{Pr}\left(\left|S_{n}\right|>\frac{1}{2} \epsilon b_{n}\right.$ i.o. $)=1$ for every $\epsilon>0$ which contradicts the condition (1).

Finally, suppose that $\sum \operatorname{Pr}\left(\left|X_{1}\right|>\epsilon b_{n}\right)<\infty$, every $\epsilon>0$. We introduce a sequence of random variables $\left\{Y_{k}, k=1,2,3, \cdots\right\}$ defined by 


$$
\begin{aligned}
Y_{k} & =X_{k} & & \text { if }\left|X_{k}\right| \leqq b_{k} \\
& =0 & & \text { if }\left|X_{k}\right|>b_{k},
\end{aligned}
$$

and write $T_{n}=\sum_{k=1}^{n} Y_{k}$. Then,

$$
\sum \operatorname{Pr}\left(Y_{k} \neq X_{k}\right)=\sum \operatorname{Pr}\left(\left|X_{1}\right|>b_{k}\right)<\infty,
$$

from which it follows that

$$
b_{n}^{-1}\left(S_{n}-T_{n}\right) \stackrel{\text { a.s. }}{\longrightarrow} 0
$$

as $n \rightarrow \infty$. Also, for $n$ sufficiently large,

$$
E Y_{n}^{2} \leqq C b_{n}^{2} \operatorname{Pr}\left(\left|X_{1}\right|>b_{n}\right),
$$

and hence $\sum b_{n}^{-2} E Y_{n}^{2}<\infty$. It then follows from a theorem of Kolmogorov (e.g. Loève $[8$, p. 238]) that

$$
b_{n}^{-1}\left(T_{n}-E T_{n}\right) \stackrel{\text { a.s. }}{\longrightarrow} 0
$$

as $n \rightarrow \infty$ and consequently, from (4), that

$$
b_{n}^{-1}\left(S_{n}-E T_{n}\right) \stackrel{\text { a.s. }}{\longrightarrow} 0,
$$

or equivalently (e.g. Loève $[8$, p. 247]),

$$
b_{n}^{-1}\left(S_{n}-\operatorname{med} S_{n}\right) \stackrel{\text { a.s. }}{\longrightarrow} 0 .
$$

This again provides a contradiction and completes the proof of the theorem.

In view of the important condition (ii) of Theorem 1, it is interesting to investigate the oscillation behaviour of sums when the summands do not satisfy condition (ii). Unfortunately, it turns out that centering complications prevent us from giving a complete answer in the general case. These complications can, of course, be avoided by working with symmetric random variables and then it is possible to give a full explanation of the behaviour. This is what we shall do in the next theorem.

Theorem 2. Suppose the $X_{i}$ are symmetric and that they do not belong to the domain of partial attraction of the normal distribution. If $\left\{b_{n}, n=1,2,3, \cdots\right\}$ is a monotone sequence of positive constants with $b_{n} \rightarrow \infty$ as $n \rightarrow \infty$, then for any $\epsilon>0, \operatorname{Pr}\left(\left|S_{n}\right|>\epsilon b_{n}\right.$ i.o. $)=0$ or 1 according as $\sum \operatorname{Pr}\left(\left|X_{1}\right|>b_{n}\right)$ converges or diverges. Equivalently, $b_{n}^{-1} S_{n}^{\text {a.s. }} \rightarrow 0$ as $n \rightarrow \infty$ if and only if $\sum \operatorname{Pr}\left(\left|X_{1}\right|>b_{n}\right)$ converges. 
The proof of this theorem involves only minor modifications of the proof of Theorem 1 and will be omitted. The result is closely related to those of Feller [3].

REMARK. By way of comparison with the result of Theorem 2, it is worth noting that under the same conditions on the $X_{i}, b_{n}^{-1} S_{n} \stackrel{p}{\rightarrow} 0$ if and only if $n \operatorname{Pr}\left(\left|X_{1}\right|>\epsilon b_{n}\right) \rightarrow 0$ as $n \rightarrow \infty$ for every $\epsilon>0$ ( " $p$ " denotes convergence in probability). This follows simply from the degenerate convergence criterion (e.g. Loève $[8, \mathrm{p} .317])$ upon making use of the Lévy condition (2).

If further structural conditions are imposed on the $X_{i}$, it is often possible to investigate the matter of centering of the $S_{n}$ process in sufficient detail to enable the symmetry assumption of Theorem 2 to be dispensed with while still obtaining the same behaviour. As an example of the possibilities, we have the following theorem.

THEOREM 3. Suppose that the $X_{i}$ belong to the domain of attraction of a nonnormal stable law of index $\alpha \neq 1$ which is not one-sided and that $E X_{i}=0$ if $E\left|X_{i}\right|<\infty$. If $\left\{b_{n}, n=1,2,3, \cdots\right\}$ is a monotone sequence of positive constants with $b_{n} \rightarrow \infty$ as $n \rightarrow \infty$, then for any $\epsilon>0$, $\operatorname{Pr}\left(\left|S_{n}\right|>\epsilon b_{n}\right.$ i.o. $)=0$ or 1 according as $\sum \operatorname{Pr}\left(\left|X_{1}\right|>b_{n}\right)$ converges or diverges. Equivalently, $b_{n}^{-1} S_{n} \stackrel{\text { a.s. }}{\rightarrow} 0$ as $n \rightarrow \infty$ if and only if $\sum \operatorname{Pr}\left(\left|X_{1}\right|>b_{n}\right)$ converges.

Proof. Again we can make use of a slightly modified version of the proof of Theorem 1 and in order to complete the proof of Theorem 3 it is just necessary to show that $b_{n}^{-1} E T_{n} \rightarrow 0$ as $n \rightarrow \infty$ where $T_{n}$ is defined as in the proof of Theorem 1.

Firstly we note that under the conditions of the theorem we may write for $x>0$, using Gnedenko and Kolmogorov [5, Theorem 2, p. 175],

$$
\operatorname{Pr}\left(\left|X_{1}\right|>x\right)=M(x) x^{-\alpha}
$$

where $M(x)$ is a function which is slowly varying as $x \rightarrow \infty$. It is necessary to treat separately the cases $\alpha<1$ and $1<\alpha<2$.

If $\alpha<1$,

$$
\begin{aligned}
\left|E Y_{n}\right| & \leqq E\left|Y_{n}\right|=\int_{|x| \leqq b_{n}}|x| d \operatorname{Pr}\left(X_{1} \leqq x\right) \\
& \leqq \int_{0}^{b_{n}} \operatorname{Pr}\left(\left|X_{1}\right|>x\right) d x
\end{aligned}
$$

using integration by parts and, using standard properties of slowly varying functions (e.g. Feller [4, p. 273]), we have 


$$
\frac{\int_{0}^{b_{n}} \operatorname{Pr}\left(\left|X_{1}\right|>x\right) d x}{b_{n} \operatorname{Pr}\left(\left|X_{1}\right|>b_{n}\right)} \rightarrow \frac{1}{1-\alpha}
$$

as $n \rightarrow \infty$ so that we can choose a constant $c_{1}>0$ such that

$$
\left|E Y_{n}\right| \leqq c_{1} b_{n} \operatorname{Pr}\left(\left|X_{1}\right|>b_{n}\right)
$$

for each $n$.

If, on the other hand, $1<\alpha<2$, we have since $E X_{1}=0$,

$$
\begin{aligned}
\left|E Y_{n}\right| & =\left|\int_{|x| \leqq b_{n}} x d \operatorname{Pr}\left(X_{1} \leqq x\right)\right| \\
& =\left|\int_{|x|>b_{n}} x d \operatorname{Pr}\left(X_{1} \leqq x\right)\right| \\
& \leqq \int_{|x|>b_{n}}|x| d \operatorname{Pr}\left(X_{1} \leqq x\right) \\
& =-\int_{b_{n}}^{\infty} x d \operatorname{Pr}\left(\left|X_{1}\right|>x\right) \\
& =b_{n} \operatorname{Pr}\left(\left|X_{1}\right|>b_{n}\right)+\int_{b_{n}}^{\infty} \operatorname{Pr}\left(\left|X_{1}\right|>x\right) d x,
\end{aligned}
$$

and

$$
\frac{\int_{b_{n}}^{\infty} \operatorname{Pr}\left(\left|X_{1}\right|>x\right) d x}{b_{n} \operatorname{Pr}\left(\left|X_{1}\right|>b_{n}\right)} \rightarrow \frac{1}{\alpha-1}
$$

as $n \rightarrow \infty$, so that we can choose a constant $c_{2}>0$ such that

$$
\left|E Y_{n}\right| \leqq c_{2} b_{n} \operatorname{Pr}\left(\left|X_{1}\right|>b_{n}\right) \text {. }
$$

Take $c=\max \left(c_{1}, c_{2}\right)$. Then, from (5) and (6) we have in general that

$$
\sum_{n=1}^{\infty} b_{n}^{-1}\left|E Y_{n}\right| \leqq c \sum_{n=1}^{\infty} \operatorname{Pr}\left(\left|X_{1}\right|>b_{n}\right)<\infty,
$$

and the result $b_{n}^{-1} E T_{n} \rightarrow 0$ follows from an application of the Kronecker Lemma to (7). This completes the sketch of the proof.

Theorems 2 and 3 provide a convenient tool for obtaining results of the type of Chover [2]. We immediately obtain from Theorem 3, for example, the following extension of the result of [2]. 
Corollary 1. Suppose that the $X_{i}$ belong to the domain of normal attraction of a nonnormal stable law of index $\alpha \neq 1$ which is not one-sided and that $E X_{i}=0$ if $E\left|X_{i}\right|<\infty$. Then,

$$
\operatorname{Pr}\left(\limsup _{n \rightarrow \infty}\left|n^{-\alpha^{-1}} S_{n}\right|^{(\log \log n)^{-1}}=e^{\alpha^{-1}}\right)=1 .
$$

(Note that in the case of normal attraction, there is a constant $c>0$ such that $\operatorname{Pr}\left(\left|X_{1}\right|>x\right) \sim c x^{-\alpha}$ as $x \rightarrow \infty$.)

\section{REFERENCES}

1. L. Breiman, Probability, Addison-Wesley, Reading, Mass., 1968.

2. J. Chover, $A$ law of the iterated logarithm for stable summands, Proc. Amer. Math. Soc. 17 (1966), 441-443.

3. W. Feller, $A$ limit theorem for random variables with infinite moments, Amer. J. Math. 68 (1946), 257-262.

4. - An introduction to probability theory and its applications. II, Wiley, New York, 1966.

5. B. V. Gnedenko and A. N. Kolmogorov, Limit distributions for sums of independent random variables, Addison-Wesley, Reading, Mass., 1954.

6. P. Hartman and A. Wintner, On the law of the iterated logarithm, Amer. J. Math. 63 (1941), 169-176.

7. P. Lévy, Théorie de l'addition des variables aléatoires, 2nd ed., Gauthier-Villars, Paris, 1954.

8. M. Loève, Probability theory, 3rd ed., Van Nostrand, New York, 1963.

9. V. Strassen, $A$ converse to the law of the iterated logatithm, Z. Wahrscheinlichkeitstheorie 4 (1966), 265-268.

Australian National University 